\title{
On the Maximum Charge State and Proton Transfer Reactivity of Peptide and Protein Ions Formed By Electrospray Ionization
}

\author{
Paul D. Schnier, Deborah S. Gross, and Evan R. Williams \\ Department of Chemistry, University of California, Berkeley, California, U.S.A.
}

\begin{abstract}
A relatively simple model for calculation of the energetics of gas-phase proton transfer reactions and the maximum charge state of multiply protonated ions formed by electrospray ionization is presented. This model is based on estimates of the intrinsic proton transfer reactivity of sites of protonation and point charge Coulomb interactions. From this model, apparent gas-phase basicities (GB ${ }^{\text {app }}$ ) of multiply protonated ions are calculated. Comparison of this value to the gas-phase basicity of the solvent from which an ion is formed enables a maximum charge state to be calculated. For 13 commonly electrosprayed proteins, our calculated maximum charge states are within an average of $6 \%$ of the experimental values reported in the literature. This indicates that the maximum charge state for proteins is determined by their gas-phase reactivity. Similar results are observed for peptides with many basic residues. For peptides with few basic residues, we find that the maximum charge state is better correlated to the charge state in solution. For low charge state ions, we find that the most basic sites Arg, Lys, and His are preferentially protonated. A significant fraction of the less basic residues Pro, Trp, and Gln are protonated in high charge state ions. The calculated $\mathrm{GB}^{\mathrm{app}}$ of individual protonation sites varies dramatically in the high charge state ions. From these values, we calculate a reduced cross section for proton transfer reactivity that is significantly lower than the Langevin collision frequency when the GB'PF' of the ion is approximately equal to the GB of the neutral base. (f Am Soc Mass Spectrom 1995, 6, 1086-1097)
\end{abstract}

E lectrospray ionization (ESI) mass spectrometry has become a powerful method to measure molecular masses of large biomolecules with high accuracy [1-3]. ESI produces distributions of multiply charged molecular ions with mass-to-charge ratios typically below 3000 , a key advantage for mass spectrometers with an upper mass range. Mechanisms for ion formation as well as explanations of charge state distributions have been hotly debated [4-9]. Factors such as biomolecule sequence $[2,10]$, conformation [5, 11-14], and solution- [15-19] and gas-phase [20-22] chemistry, as well as instrumental response, influence these charge distributions. For example, proteins electrosprayed from "native" solutions have charge distributions shifted to lower charge (higher mass-to-charge ratio) than those formed from denaturing solutions [11, 12]. Reduction of disulfide bonds in disulfide-containing proteins also results in a shift of the charge state distribution to lower mass-to-charge ratio $[13,14]$. Fenn has proposed a model that qualitatively accounts for these, as well as other observations, based on the charge density on the surface of the evaporating droplet [5].

Address reprint requests to Professor Evan R. Williams, Department of Chemistry, University of California, Berkeley, Berkeley, CA 94720).
A common approach for estimation of the maximum extent of protonation for a protein or peptide electrosprayed from acidic solution was proposed by Covey et al. [10], who found that the maximum charge state was often similar to the number of basic sites (Arg, Lys, His, and $\mathrm{N}$-terminus). These sites are protonated in the acidic solutions commonly used in ESI. In 1991, Smith et al. [2] compiled a list of proteins and peptides for which ESI spectra had been obtained. This list includes the number of basic residues and the maximum charge state observed for each of these molecules. Excellent agreement between the maximum charge state and the number of basic residues is observed for many of these molecules. However, for several of these molecules, significant deviations are observed. For example, S4 ribosomal protein has 46 basic sites, yet the highest charge state reported in the literature for this molecule is $30+$ [23]. Actin has 46 basic residues, but charge states as high as $59+$ have been reported [2]. In some molecules, not all of these basic sites are protonated. In others, additional residues must be protonated.

Aqueous solution-phase equilibria models have been proposed to explain the charge distributions seen in ESI [15-19]. However, significant deviations between solution-phase and gas-phase charge distributions have been reported $[15-18,20,21]$. For example, 
positive ESI spectra of proteins have been observed from basic solutions in which these ions have a net negative charge [15-17]. Wang and Cole [21] found that the ratio of $\left[\mathrm{M}+2 \mathrm{H}^{2+}\right] /\left[\mathrm{M}+\mathrm{H}^{+}\right]$for both gramicidin $\mathrm{S}$ and bradykinin did not change significantly when the $\mathrm{pH}$ of the solution was varied from $\sim 3$ to 11 . Over this $\mathrm{pH}$ range, the calculated ratio for these ions in solution changes from $10^{5}$ to $10^{-2}$ for gramicidin $\mathrm{S}$ and from $10^{6}$ to $10^{-2}$ and for bradykinin. LeBlanc et al. [20] also found that changes in this ratio for gramicidin $\mathrm{S}$ did not correlate with solution $\mathrm{pH}$, but found this ratio decreased with increasing proton affinity of nitrogen-containing bases added to the solution. These results indicate that gas-phase chemistry influences the charge state distributions of these ions.

Proton transfer reactions of multiply protonated ions formed by ESI have been investigated by a number of groups. McLuckey et al. [24] measured the rates of proton transfer from isolated charge states of cytochrome $c$ with neutral dimethylamine and found these rates decreased with lower charge. Cassady et al. [25] reported similar results for isolated charge states of ubiquitin. Smith and co-workers $[13,14,22,26,27]$ have investigated the proton transfer reactions of a variety of proteins with neutral gas-phase bases in the interface region of the ESI source and found that the charge state distributions shift to lower charge upon addition of bases with increasing proton affinity.

These studies indicate that the distribution of multiply protonated ions observed in ESI can be significantly altered by gas-phase chemistry. We have reported a model that quantitatively accounts for the proton transfer reactions of multiply protonated ions, which include doubly protonated gramicidin S [28] and diaminoalkanes [29], as well as individual charge states of cytochrome $c$ [30] and lysozyme [31]. Results for both these proteins indicate that the maximum charge state for these ions formed from both "native" and denaturing solutions can be explained based on the relative apparent gas-phase basicity of the multiply protonated ion and that of the solvent molecules from which these ions are electrosprayed. We present here the extension of our model to a variety of peptides and proteins as large as bovine albumin with 95 charges, and we show that our calculations based on gas-phase chemistry provide a method to determine the maximum charge state of peptide and protein ions formed by ESI with improved accuracy.

\section{Method}

\section{Apparent Gas-Phase Basicity}

The gas-phase basicity (GB) of a neutral molecule, $M$, is defined as the negative Gibbs free energy for eq 1:

$$
\mathrm{M}+\mathrm{H}^{+} \leftrightarrow \mathrm{MH}^{+} \quad \mathrm{GB}(\mathrm{M})=-\Delta G_{1}
$$

A variety of experimental methods have been developed to measure this value [32-34]. In contrast, measurement of the GB of an $\mathrm{MH}_{n}^{n+}$ ion is more complicated because of the presence of an activation barrier due to Coulomb interaction of two charged ions [35, 36] (eq 2).

$$
\mathrm{MH}^{+}+\mathrm{H}^{+} \leftrightarrow \mathrm{MH}_{2}^{2+} \quad \mathrm{GB}\left(\mathrm{MH}^{+}\right)=-\Delta G_{2}
$$

From measurements of proton transfer reactions of multiply protonated ions, the apparent or kinetic gasphase basicity (GB ${ }^{\text {apF}}$ ) of protonated ions can be obtained. This value has been measured for a variety of species, which include $\mathrm{C}_{6(1)} \mathrm{H}^{\cdot 2+}$ [37], gramicidin $\mathrm{S}$ [28], diaminoalkanes [29], and multiply protonated proteins $[30,31]$ by using the bracketing method $[32,33]$ in which rates of proton transfer from multiply charged ions to neutral reference bases of known GB are measured. The true GB of these charged species is lower than the $G B^{\text {app }}$ by a value equal to the reverse activation barrier for the separating charged products.

We have proposed that the proton transfer reactivity of multiply charged ions can be accounted for from estimates of the intrinsic proton transfer reactivity of a protonation site $t\left(G B_{\text {Intrinsic, }}\right)$ and the Coulomb energy experienced by this site from other protons (eq 3 ) [28-31],

$$
\mathrm{GB}^{\mathrm{app}}=\mathrm{GB}_{\text {Intrinsic, } 1}-\sum_{i=1}^{\prime \prime} \frac{q^{2}}{\left(4 \pi \varepsilon_{0}\right) \varepsilon_{r} r_{i, 1}}
$$

where $\varepsilon_{r}$ is the effective shielding between charges in these gas-phase ions. From measured proton transfer reactions of doubly protonated diaminoalkanes [29] and gramicidin $S$ [28], values of $\varepsilon_{r}=1.01 \pm 0.07$ and $<1.2$, respectively, were obtained. In contrast, the measured GB.pp of individual charge states of the proteins cytochrome $c$ [30] and lysozyme [31] could be fitted to eq 3 by using a value of $\varepsilon_{r}=2.0$. The significance of these values will be discussed in subsequent text.

\section{Calculated Lowest Energy Proton Configurations}

To calculate the GBapp of a site of protonation in a protein from eq 3, information about where charges reside in the protein must be obtained. McLafferty and co-workers $[38,39]$ found that cytochrome $c$ ions with as many as 16 protons undergo up to $133 \mathrm{H} / \mathrm{D}$ exchanges with $\mathrm{D}_{2} \mathrm{O}$. The rates of these reactions fit pseudo-first order kinetics with a single rate constant. Because exchange is primarily expected to occur at charge sites, these results indicate that intramolecular transfer of $H / D$ to various sites in the ion occurs faster than the intermolecular $H / D$ exchange rate. Protons also are expected to sample different sites in the protein. Thus, under near thermal reaction conditions, a population of the lowest free energy charge configurations should be present. However, in the ESI process, 
ions with excess internal as well as kinetic energy can be produced. These higher energy ions will be more reactive toward proton transfer. Thus, the highest charge states should primarily consist of ions with low internal and kinetic energy.

To find these lowest energy charge configurations, we calculate a relative free energy of a given charge configuration via eq 4 :

$$
\sum_{\substack{i, j \\ i>i}}^{n} \frac{q^{2}}{\left(4 \pi \varepsilon_{0}\right) \varepsilon_{r} r_{i, i}}-\sum_{i=1}^{n} \mathrm{~GB}_{\text {Intrinsic, }}
$$

where the first term is the total Coulomb energy in the ion and the second term is the sum of $G B_{\text {Intrinsic of }}$ the sites of protonation. Proteins and peptides without disulfide bonds are modeled as an elongated onedimensional "string" with residues separated by $3.8 \AA$ [40]. This separation corresponds to the maximum distance possible between amino acid residues in the gas phase and thus minimizes the calculated Coulomb repulsion. It is expected that for very high charge states, a highly elongated ion conformation will be significantly favored. Our results do not directly depend on this value of $3.8 \AA$; a smaller separation distance could be modeled with a higher effective shielding value $\left(\varepsilon_{r}\right)$ to give identical results, that is, our assumed ion conformation and our $\varepsilon_{r}$ are coupled. Proteins with disulfide bonds and those electrosprayed from a solution in which the protein is in a native conformation are modeled by using the x-ray crystal coordinates of the basic side chain nitrogens for the basic amino acids and the backbone carbonyl oxygens for all other residues. This model differs from the model of Rockwood et al. [41], who calculated the effects of charge on dissociation energies, in that our model incorporates the biomolecule sequence, the effective shielding between charges, and the ion conformation.

The values of $G B_{\text {Intrinsic }}$ of various sites in a protein are poorly understood. In the gas phase, intramolecular solvation of charge can occur, which results in increased stabilization of the charges and increased GB [42-44]. This charge solvation is energetically favorable, even in the very highly protonated ions formed by ESI (vide infra). We crudely estimate these values for Pro, Trp, GIn, and Lys to be 234.3, 234.3, 237.4, and $241.0 \mathrm{kcal} / \mathrm{mol}$, respectively, based on measurements of the GB of small peptides that contain only one of these basic residues [30]. Proline is included as a basic side-chain residue even though the side-chain nitrogen is present as an amide in a peptide chain. Amster and co-workers [45] reported values of GB for the dipeptides VV, PV, and VP to be $214.8 \pm 3.1,221.9 \pm 2.9$, and $223.7 \pm 1.9 \mathrm{kcal} / \mathrm{mol}$, respectively. The higher basicity of VP versus PV, both of which are significantly higher than VV, indicates that proline is a basic site in a peptide even in an amide form. The $\mathrm{GB}_{\text {Intrinsic }}$ of Arg and His are assigned values of 251.3 and 244.8 $\mathrm{kcal} / \mathrm{mol}$, respectively, and correspond to the GB of the individual amino acids [46-48] plus $15 \mathrm{kcal} / \mathrm{mol}$; the latter value is an estimate of the intrinsic stabilization energy. For all other residues and the $N$-terminus, we use $\mathrm{GB}_{\text {lntrinsic }}=221.6 \mathrm{kcal} / \mathrm{mol}$, which corresponds to the estimated value for protonation of the backbone [49] plus $15 \mathrm{kcal} / \mathrm{mol}$. Clearly, these values are rough estimates of the $G B_{\text {Intrinsic }}$ of sites of protonation in a protein. More accurate values will be obtained through investigation of additional model peptides and through a better understanding of the local interactions associated with protonation of various sites in a protein.

For a protein with $r$ residues and $n$ charges, the number of possible charge configurations is given by eq 5:

$$
\text { \# charge configurations }=\frac{\prod_{i=0}^{(11-1)}(r-i)}{n !}
$$

For a small protein such as ubiquitin, which has 76 residues, there are $\sim 10^{1.4}$ ways to assign 13 protons, the maximum charge state reported for this protein [50]. This number of configurations is sufficiently small that the relative free energy of each of these configurations can be calculated (approximately four days on a dedicated IBM RS/6000). For larger proteins, a direct calculation of the energy of every configuration is not feasible due to both the larger number of configurations and the increased time required to calculate the Coulomb term for each configuration. For example, the number of proton configurations for the $45+$ charge state of carbonic anhydrase ( 260 residues) is $-10^{\text {51) }}$. To calculate the energy of every possible charge configuration explicitly would take $>10^{40}$ years.

To circumvent this problem, we have developed a "pseudo-random walk" algorithm to find the lowest free energy charge configurations for larger proteins. An initial version of the algorithm was developed specifically for cytochrome $c$ and these results are reported elsewhere [30]. We have modified this algorithm to find the lowest energy charge configurations for a wide range of peptides and proteins with molecular masses up to $100 \mathrm{kDa}$. To begin, n charges are assigned to individual residues in a protein such that they are spread evenly along the molecule. The relative free energy of this charge configuration is calculated using eq 4. A single charge is then randomly selected and moved either to an unoccupied basic side chain residue (Arg, Lys, His, Pro, Gln, and Trp) or placed at an unoccupied backbone site that is within 20 residues of its current location. In the former process, we move the proton to a basic site within 20 residues of its current location with a $95 \%$ probability and to any basic site in the entire molecule with a $5 \%$ probability. In the latter process, the backbone site is selected so as to minimize the Coulomb energy and hence the total energy of the ion. Different probabili- 
ties are assigned to these two processes and are changed as a function of $n$. When $n$ is less than half the number of residues with basic side chains (Arg, Lys, His, Pro, Gln, and Trp) in the protein, we assign the probability of these moves to be $95 \%$ and $5 \%$, respectively. For larger $n$, we increase the probability of moving a charge to a backbone site linearly until the probability of either move is equal; this occurs when $n$ is equal to the number of basic side-chain sites. These probabilities are evaluated empirically and reflect the greater likelihood of protonation of basic side-chain sites for low $n$ and backbone sites with increasing $n$.

The free energy of this new configuration is calculated. If this value is less than that of the previous charge configuration, then this new configuration is "accepted" and this process is repeated. If the free energy of this charge configuration is greater than that of the previous one, we "accept" this new charge configuration with a probability given by eq 6 :

$$
\text { probability }=-\frac{1}{N} \Delta E_{T}+1
$$

where $\Delta E_{T}$ is the increase in free energy of the new ion configuration (a negative probability is assigned a value of 0 ). $N$ is assigned a value of either 16 or 50 with a probability of 0.9 and 0.1 , respectively. The values of 16 and 50 enable a proton configuration that is 16 and $50 \mathrm{kcal} / \mathrm{mol}$ higher to be accepted. The former value corresponds to a difference in energy required to move a proton from either Arg to Pro/Trp or from Pro/Trp to a backbone site (excluding Coulomb energy). The latter value is the energy required to move a proton from Arg to a backbone site. This allows protons to have some small but finite probability of being moved relatively freely along the protein. This makes it possible to traverse barriers of any significance that are likely present on the multidimensional potential energy surface and to avoid configurations that are trapped in a local minimum.

This entire process is repeated until no new lowest energy configurations are found for at least $10^{4}$ iterations. To avoid being trapped in an area of the potential energy surface isolated by barriers $>50 \mathrm{kcal} / \mathrm{mol}$, the charges are then "scrambled" by randomly moving each charge. New lowest energy configurations are then searched out and the entire process is repeated between $10^{2}$ and $10^{3}$ times dependent on 11 . The process is repeated $10^{2}$ times for $n<15,5 \times 10^{2}$ times for $n=15-30$, and $10^{3}$ times for $n>30$.

\section{Testing}

Standard searching algorithms [51-53] that search out minima by sampling only a relatively small number of points on a complex surface typically find the global minimum less than $10 \%$ of the time [53]. The minima searching algorithm employed in these calculations is expected to have a higher success rate than algorithms for a generic surface because we know a priori that most of the possible proton configurations are very high in energy. Configurations in which the charges are significantly separated but still are located primarily at the most basic sites will be favored. Thus, our algorithm does not work efficiently to find the absolute minimum for molecules in which the $G_{\text {Intrinsic }}$ of all sites are similar. This could be improved by giving a greater weight to the Coulomb term in such molecules.

Tests of our previous algorithm with cytochrome $c$, which has 104 residues, indicated that the lowest energy configurations were found [30]. Because the number of configurations increases dramatically with protein size and $n$, additional testing on the $45+$ charge state of carbonic anhydrase was performed. Initial charge configurations were varied by placement of all charges on adjacent sites on the $N$-terminus, the $C$ terminus, and in the middle of the protein. In each case, the same lowest energy configurations were found. To determine whether the number of iterations is sufficient for convergence, this number was increased by 50 . No additional charge configurations were found within $3 \mathrm{kcal} / \mathrm{mol}$ of the lowest energy configuration. These results indicate that the lowest energy proton configurations are found. We continue to test this algorithm to determine if we truly are finding the global minimum. We also are investigating modifications that improve its efficiency for finding the lowest energy charge configurations for large ions.

\section{Calculated $G B^{a p n}$}

To determine the GBapp of a given charge configuration, the $G B^{\text {NPP }}$ of each proton in a given configuration is calculated from eq 3 . The GB ${ }^{\mathrm{F} F}$ of this configuration is assigned that of the proton with the lowest GB ${ }^{\text {ipp. }}$ For most of the charge states of a protein, numerous proton configurations with energies within a few $\mathrm{kcal} / \mathrm{mol}$ of the lowest energy configuration are found. For example, we find that there are 17 charge configurations of the 46 + charge state of carbonic anhydrase that are within $3 \mathrm{kcal} / \mathrm{mol}$ of the lowest energy configuration. Some fraction of each of these configurations should be present. We approximate this ion population by assumption of an equal probability of each of these charge configurations. The GBapf of each charge state is thus assigned the average of the GB $\mathrm{Bp}$ of all the charge configurations within $3 \mathrm{kcal} / \mathrm{mol}$ of the lowest free energy configuration.

\section{Maximum Charge State}

By comparison of our calculated GB.pp to the GB of the solvent, we determine the highest charge state of an ion that would not undergo proton transfer to solvent molecules in the gas phase. That is, it is energetically and kinetically favorable for an ion with a GBapp below that of the solvent to undergo proton transfer to the solvent to form protonated solvent and an ion with 
one less charge. Thus, we determine the maximum charge state to be that of the first charge state with a calculated $G^{\text {app }}$ below the GB of the solvent. For solutions that have more than one component, for example, methanol, water, and acetic acid, we use the GB of the most basic component, in this case, methanol. This mechanism is useful to explain the maximum possible extent of protonation on an ion formed by ESI. This is not intended to account for the charge distributions typically observed in an ESI mass spectrum nor does it address solution-phase chemistry. Effects of the latter on the maximum charge state are discussed in subsequent text.

\section{Results}

\section{Maximum Charge State}

The calculated $\mathrm{GB}^{\mathrm{app}}$ as a function of charge state for 10 molecules is shown in Figure 1. The maximum experimental charge state reported in the literature for each molecule, electrosprayed from methanolcontaining solutions, is circled. The calculated GB ${ }^{\text {app }}$ for each of these maximum charge states is close to the $G B$ of methanol $(G B=174.1 \mathrm{kcal} / \mathrm{mol}$ [48]), which indicates a reasonable correlation between the maximum charge state on an ion and the GB of the solvent. For larger proteins, such as bovine serum albumin, the difference in calculated GB ${ }^{\text {app }}$ between adjacent charge states is small. Therefore, we expect lower absolute accuracy for calculation of the maximum charge state for these large ions.

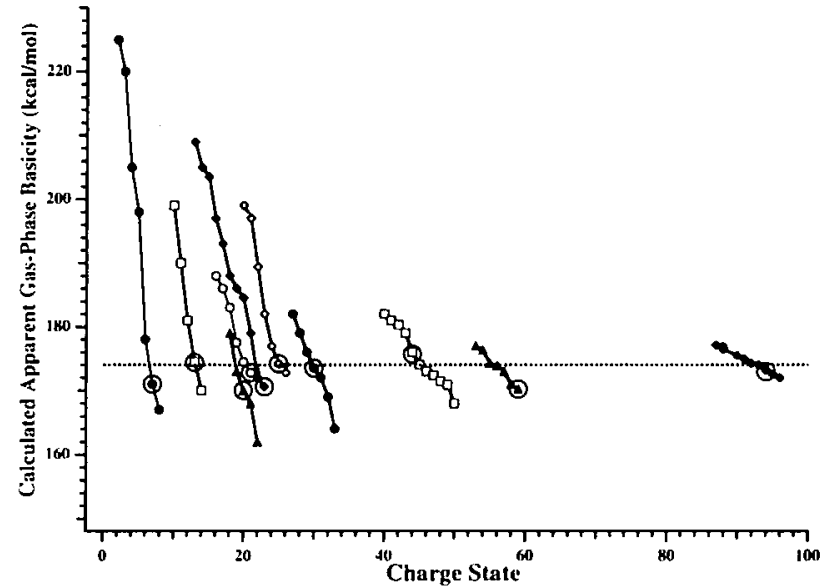

Figure 1. Calculated apparent gas-phase basicity as a function of charge state for nine molecules modeled as elongated strings by using an $\varepsilon,=2.0$. In order from left to right: melittin (O); ubiquitin ( $\square$ ); cytochrome i (tuna) ( $\Delta$ ); cytochrome i (equine) $(O)$; ribonuclease A $(\$)$; hemoglobin ( $\alpha$-chain) ( $)$; myoglobin (O); carbonic anhydrase ( $\square$ ); actin ( $\Delta$ ); bovine serum albumin ( ). The dashed line indicates GB of methanol $(174.1 \mathrm{kcal} / \mathrm{mol})$; circled values correspond to maximum experimental charge states reported in the literature for each protein.

The maximum charge state obtained from our calculated $\mathrm{GB}^{\text {app }}$ values for a variety of peptides and proteins is given in Tables 1-3. Also reported in these tables are the number of basic sites and the maximum experimental charge state that has been reported in the literature, to our knowledge, for these molecules. It is important to emphasize that ESI charge distributions depend on a number of factors (vide supra). Not all

Table 1. Maximum charge states of 15 peptides electrosprayed from methanol-containing solutions"

\begin{tabular}{|c|c|c|c|c|c|}
\hline \multirow[b]{2}{*}{ Peptide } & \multirow{2}{*}{$\begin{array}{c}M W \\
\text { (u) }\end{array}$} & \multirow{2}{*}{$\begin{array}{c}\text { Basic } \\
\text { residues }^{b}\end{array}$} & \multicolumn{2}{|c|}{ Maximum charge state } & \multirow[b]{2}{*}{$\varepsilon$, Range } \\
\hline & & & Experimental & Calculated $^{c}$ & \\
\hline YYYYYY & 997 & 1 & 1 & $2^{\dagger}$ & \\
\hline Angiotensin II & 1046 & 3 & 3 & 3 & $1.0-1.4$ \\
\hline Bradykinin & 1060 & 3 & 3 & 3 & $1.2-1.6$ \\
\hline DRVYIHPFHLLVYS & 1759 & 4 & $4^{\mathrm{d}}$ & 4 & $1.0-2.1$ \\
\hline IIFWGGPGSGKGTOCEK & 1853 & 3 & 3 & $4^{\dagger}$ & \\
\hline STEKLKHHKIIFWGG & 1902 & 5 & 4 & 4 & $1.0-1.6$ \\
\hline SLAMMAASDIDLLDAQSAPLR & 2032 & 2 & 2 & $4^{\dagger}$ & \\
\hline LTDQQAPPHQDCAC & 2072 & 2 & 2 & $3^{\dagger}$ & \\
\hline$(\mathrm{GHHPH})_{5} \mathrm{G}$ & 2094 & 16 & 6 & 6 & $1.3-1.6$ \\
\hline Dynorphin A (porcine $1-17$ ) & 2147 & 6 & 4 & 4 & $1.3-1.8$ \\
\hline PPPPEEROETRSOTPAPKPS & 2229 & 4 & 4 & 5 & $1.0-1.2$ \\
\hline Melittin & 2845 & 6 & 7 & 6 & $1.6-2.1$ \\
\hline KGFIORISKGGLIKTKRKRKKORVK & 2981 & 13 & 9 & 8 & $1.5-1.8$ \\
\hline GNRGRGQRRDWSRAPPPPEERQETRS & 3076 & 8 & 6 & 6 & $1.1-1.3$ \\
\hline Calcitonin (human) & 3418 & 3 & 3 & 3 & $1.2-1.5$ \\
\hline
\end{tabular}

\footnotetext{
Experimental values are obtained from ref 2 . Calculated values are obtained with an $\%$, 1.3 and a linear conformation. The range of $\theta$, for which the experimental maximum charge state is correctly calculated is reported.

${ }^{b}$ Sum of Arg, Lys, His, and $N$-terminus.

c Peptides for which the maximum charge state corresponds to the charge state in solution are indicated by $t$

Electrosprayed from acetonitrile $(\mathrm{GB}=180 \mathrm{kcal} / \mathrm{mol})$.
} 
Table 2. Maximum charge states of commonly electrosprayed proteins (five or more published spectra)"

\begin{tabular}{|c|c|c|c|c|c|}
\hline \multirow[b]{2}{*}{ Protein } & \multirow{2}{*}{$\begin{array}{l}M W \\
\text { (u) }\end{array}$} & \multirow{2}{*}{$\begin{array}{c}\text { Basic } \\
\text { residues }^{b}\end{array}$} & \multicolumn{2}{|c|}{ Maximum charge state } & \multirow[b]{2}{*}{$\varepsilon_{r}$ Range } \\
\hline & & & Experimental & Calculated & \\
\hline Ubiquitin (bovine) & 8,565 & 13 & $13^{\mathrm{c}}$ & 14 & $1.7-2.2$ \\
\hline Cytochrome $c$ (equine, denatured) & 12,358 & 25 & 21 & 21 & $1.9-2.2$ \\
\hline Cytochrome $c$ (equine, native) & 12,358 & 25 & $11^{\mathrm{d}}$ & 11 & \\
\hline Cytochrome $c$ (tuna) & 12,029 & 20 & 20 & 19 & $1.8-2.1$ \\
\hline Ribonuclease A (denatured) & 13.682 & 19 & 23 & 22 & $1.9-2.3$ \\
\hline Ribonuclease A (disulfide-intact) & 13,682 & 19 & 15 & 11 & \\
\hline Lysozyme (denatured) & 14,306 & 19 & 22 & 22 & $1.8-2.2$ \\
\hline Lysozyme (disulfide-intact) & 14,306 & 19 & 14 & 14 & \\
\hline Hemoglobin ( $\alpha$-chain) & 15,126 & 25 & 25 & 26 & $2.2-2.4$ \\
\hline Myoglobin (equine) & 16.951 & 33 & $30^{\prime}$ & 30 & $1.9-2.3$ \\
\hline$\beta$-Lactoglobulin B (bovine) & 18.277 & 21 & 19 & 24 & $1.6-1.8$ \\
\hline Carbonic anhydrase (bovine) & 29.021 & 37 & $44^{9}$ & 45 & $1.7-1.9$ \\
\hline Carbonic anhydrase (human) & 29,157 & 38 & 38 & 42 & $2.2-2.5$ \\
\hline Serum albumin (bovine, denatured) & 66.430 & 100 & $-90-95$ & 95 & $2.1-2.3$ \\
\hline
\end{tabular}

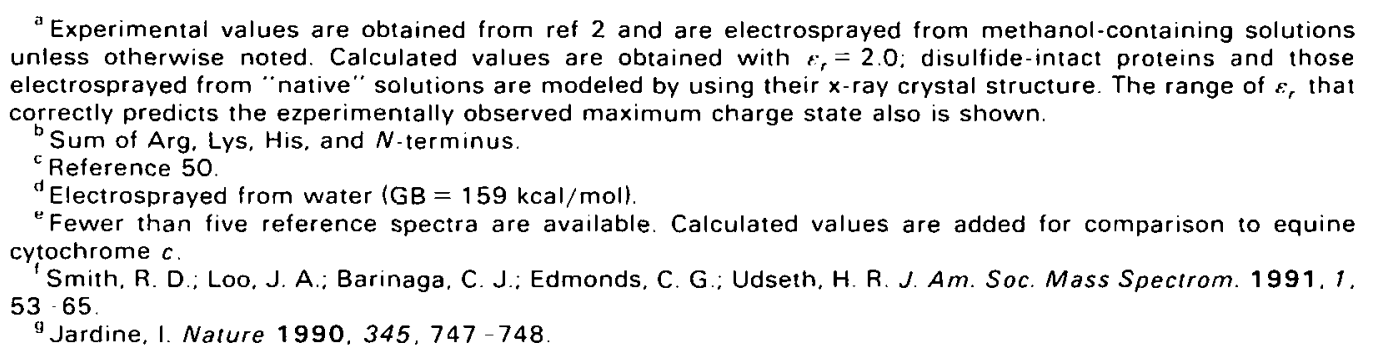

spectra reported in the literature have the maximum charge state reported in the table. In addition, we may have inadvertently missed some spectra that have higher charge states. To partially compensate for this, we have separated the proteins into two categories. Table 2 contains commonly measured proteins for which five or more spectra have been published. In many cases, such as equine cytochrome $c$, this number is significantly greater. Proteins with four or fewer spectra are reported in Table 3 . These latter proteins were selected arbitrarily from ref 2 . We expect that there is a greater probability that the highest possible charge state has been reported for proteins (electrosprayed from a particular solvent system) for which a large number of spectra have been reported. For proteins in Table 3, our calculated values tend to overestimate the maximum charge state more frequently and to a greater extent than for proteins in Table 2 . This indicates that the "true" maximum charge state may not have been observed yet for these proteins.

Table 3. Maximum charge states of nine arbitrarily selected proteins for which there are four or fewer published spectra, electrosprayed from methanol-containing solutions unless otherwise noted

\begin{tabular}{lcccc}
\hline Protein & $\begin{array}{c}\text { MW } \\
(\mathrm{u})\end{array}$ & $\begin{array}{c}\text { Basic } \\
\text { residues }^{\text {a }}\end{array}$ & Experimental & Calculated \\
\hline \hline Interleukin-2 (recombinant) & 15.416 & 19 & 15 & 17 \\
Ecotin & 16.097 & 22 & $21^{\text {b }}$ & 21 \\
Myoglobin (sheep) & 16.923 & 27 & 26 & 28 \\
Mouse urine protein (male) & 18,883 & 27 & 19 & 23 \\
S4 ribosomal protein & 21,138 & 46 & 30 & 34 \\
Human growth hormone & 22,125 & 24 & $23^{\text {c }}$ & 26 \\
Pepsin (porcine) & 34,510 & 5 & $11^{\text {d }}$ & 40 \\
Actin & 41.862 & 46 & 59 & 56 \\
HIV transcriptase & 51,302 & 87 & & 49 \\
\hline
\end{tabular}

Sum of Arg. Lys, His, and N-terminus.

bibson. B. W. In Techniques in Protein Chemistry 11; Villafranca. J. J., Ed.; San Diego. CA: Academic Press, 1991; pp $419-425$.

Clectrosprayed from water (GB $=159 \mathrm{kcal} / \mathrm{mol})$

deference 55 


\section{Dielectric Polarizability}

Values of $\varepsilon_{r}=1.3$ and 2.0 are used to calculate the maximum charge states for all peptides and all proteins, respectively. The latter value is obtained from a best fit of calculated $G^{\text {app }}$ values to those experimentally measured for the $3+$ to $15+$ charge states of cytochrome $c$ [30]. To show the effect of $\varepsilon_{r}$ on our calculation, the range of $\varepsilon_{r}$ that can be used to obtain the experimentally observed maximum charge state is also given in Tables 1 and 2 . The range of $\varepsilon_{r}$ that correctly matches the experimental value for peptides $(1.0-2.1)$ is lower than that for proteins $(1.6-2.5)$. Although these ranges overlap, peptides appear to have less effective shielding between charges than proteins. An alternate possibility is that the barrier to proton transfer is a larger fraction of the "true" coulomb energy in these ions. This is consistent with our previous results, which indicate a value of $\varepsilon_{r}<1.2$ for gramicidin $S$ [28] and $\varepsilon_{r}=2.0$ for cytochrome $c$ [30] and lysozyme [31]. Remarkably, a single value $\left(\varepsilon_{r}=\right.$ 2.0) appears to be effective to determine the maximum charge state for a wide variety of proteins. Similarly, one value $\left(\varepsilon_{r}=1.3\right)$ is effective for peptides. We continue to investigate the relationship of molecular size, functional groups, ion structure, and the potential surface for proton transfer to this value.

\section{Discussion}

\section{Gas-Phase Versus Solution Chemistry}

For molecules in which the number of basic residues in the peptide or protein exceeds the number of charge states observed experimentally, our calculations based on the gas-phase chemistry are quite accurate. For example, the peptide $(\mathrm{GHHPH})_{5} \mathrm{G}$ has 16 basic residues, each of which is expected to be protonated in acidic solution. The maximum experimental charge state is only $6+$. This value is correctly predicted from our calculations. For the peptide KGFIQRISKGGLIKTKRKRKKQRVK, we calculate a maximum charge state of 8 . This peptide has 13 basic sites, but $9+$ is the maximum charge state observed experimentally [2]. Similar results are observed for proteins. Bovine albumin has 100 basic residues, but only $\sim 90-95$ charges are observed experimentally. We calculate this value to be 95 , a reasonable agreement with the experimental value. The higher number of positive charges in solution than the gas phase is directly attributable to differences in the shielding provided by the surrounding medium. For water, methanol, and vacuum these values are reflected by the dielectric constants of these media $(79,33$, and 1, respectively [54]).

For peptides with few basic sites, we find that our calculations overestimate the maximum number of charges. This occurs for 5 of the 15 peptides in Table 1. For four of these peptides, the maximum charge state can be readily explained by the maximum extent of protonation in solution (based on the number of basic residues). For example, our calculations indicate that the peptide, $(Y)_{0}$, protonated on the $N$-terminal and $C$-terminal residues has a $\mathrm{GB}^{\mathrm{app}}$ of $203 \mathrm{kcal} / \mathrm{mol}$, which is $29 \mathrm{kcal} / \mathrm{mol}$ greater than the GB of methanol (GB = $174.1 \mathrm{kcal} / \mathrm{mol}$ ). Thus, if this ion were formed, our calculations indicate that it should not undergo proton transfer to methanol under thermal reaction conditions. However, only the $\mathrm{N}$-terminus is protonated in solution. This indicates that the maximum charge state of this peptide is determined by its maximum charge state in solution. The maximum charge state can be explained based on the maximum solution charge state for four of the five peptides for which our calculations based on gas-phase chemistry overestimate this value.

In striking contrast, for proteins the number of basic residues protonated in solution appears to play less of a predictive role for the maximum charge state than our calculated GB ${ }^{\mathrm{PP}}$. For example, actin has only 46 basic sites, yet the maximum experimental charge state is $59+$. Our calculations indicate a maximum charge state of $56+$. It has been suggested that charge states greater than the number of basic residues could be formed by gas-phase protonation [17]. However, this is unlikely due to the significant Coulomb barrier to bring two like charged products together. We calculate the barrier for this process to be $64 \mathrm{kcal} / \mathrm{mol}$ for the $56+$ ion of actin. Even if this energy is accessible in the interface region of an ESI source, the impact parameter for this process should be exceedingly small due to the long range $1 / r$ ( $r$ is the intercharge separation) dependence of this interaction.

We believe a more likely explanation is that the maximum charge state protein ions are formed through a process in which there is a significant clustering of solvent with the ion; the isolated ion is subsequently formed by solvent evaporation from the charged ion-solvent cluster. Such a cluster could carry more charge than the bulk solution charge on a protein ion and also more than that of an isolated protein ion due to the stabilization of solvated charges in a high dielectric solvent (the Born energy). As solvent evaporation occurs, the charge on the cluster would be expected to partition between the departing solvent molecules and the remaining protein ion. Toward the end of this process, the protein-solvent complex would be expected to reflect the gas-phase chemistry of these species. The initial solvent-protein ion cluster could be formed from either a charge residue process [6] or from an Iribarne and Thomson ion evaporation process [8] in which the protein ion is desorbed from a charged droplet with a significant number of solvent molecules attached. For the latter process, desorption of a solvated ion should be more energetically favorable than desorption of an isolated ion, particularly for larger molecules with many charges. The correlation between solution-phase charge state and maximum experimental charge state for peptides with few basic residues suggests that smaller peptides are primarily desorbed 
from a droplet with significantly less solvation than for proteins.

One clear exception to the predictive capabilities of our model is the 326 residue protein pepsin, which has only five basic sites. We calculate that the $40+$ ion should be stable with respect to proton transfer to methanol. Very recently, Standing and co-workers [55] reported an ESI spectrum for this molecule with up to $11+$ charges. These results are consistent with an ion evaporation model in which a solvated ion cluster, desorbed from a droplet, carries a net charge greater than the solution-phase charge state of the protein, but less than the total number of charges that the protein can retain in the gas phase. In contrast, we expect a maximum charge state of $\sim 40+$ should be observed if these ions are formed by a pure charge residue process, because significantly more than $40+$ charges should be available to partition between the protein and the departing solvent molecules.

\section{Ion Conformation}

The conformation of a protein in solution can effect the charge distribution and maximum charge state in the electrospray ionization mass spectrum [5, 11-13]. Proteins electrosprayed from solutions in which the protein is in a native conformation have charge distributions shifted to higher mass-to-charge ratio (lower charge) than their denatured counterparts [11-14]. For example, equine cytochrome $i$ electrosprayed from pure water has a maximum charge state of $11+;$ when it is electrosprayed from denaturing acidic watermethanol solution, a maximum charge state of $21+$ is observed [2]. Because electrostatic forces are long range interactions, the Coulomb energy in an ion will depend strongly on the distance between charges and hence the ion conformation. Thus, conformation will influence the GBapp of an ion. When cytochrome $i$ is modeled in its crystal structure, we calculate the GB" of the $11+$ charge state, which indicates that the proton transfer reactivity of the $12+$ charge state (eq 2) is $156 \mathrm{kcal} / \mathrm{mol}$. This value is slightly below the GB of water $(159 \mathrm{kcal} / \mathrm{mol})$. Thus, we would expect the $11+$ ion to be the maximum charge state for an ion that is in its native conformation. This agrees with the experimentally observed value.

For lysozyme, the maximum experimental charge states are $22+[14]$ and $14+$ [56] for the disulfidereduced and disulfide-intact proteins, respectively, electrosprayed from acidified aqueous solutions that contain methanol. We calculate $23+$ and $14+$ by using a linear and crystal structure, respectively. The agreement between the experimental and calculated values for the disulfide-intact protein indicates that lysozyme retains a folded conformation even in an acidified methanol-containing solution. Dobson and co-workers [57] have shown that lysozyme is in its native conformation in an aqueous trifluoroethanol $(15 \% \mathrm{v} / \mathrm{v})$ solution at $\mathrm{pH}=2.0$ and remains folded even when the trifluoroethanol content is increased to $50 \%(\mathrm{v} / \mathrm{v})$, although this new structure is different from the native conformation. In the solution from which the maximum charge state of the disulfide-intact protein is observed [56], it is unlikely that the protein is in its native conformation. However, the agreement between our calculations using the crystal structure and the experimentally observed maximum charge state indicates that lysozyme remains in a compact form even in this solution.

For ribonuclease $\mathrm{A}$, the calculated values are $22+$ and $11+$ using a denatured and crystal structure, respectively. Experimental values of $23+$ and $15+$ have been reported [2] for the disulfide-reduced and disulfide-intact protein electrosprayed from acidified methanol-containing solutions. The high negative deviation in our calculated values for the disulfide-intact protein via the crystal structure indicates that these ions are partially unfolded in solution. The transition temperature $\left(T_{m}\right)$ from the native to the unfolded conformation of ribonuclease $\mathrm{A}$ is $-312 \mathrm{~K}$ at $\mathrm{pH}=2.0$ (glycine- $\mathrm{HCl}$ buffer) [58]. Under these same conditions, the $T_{m}$ of lysozyme is $12 \mathrm{~K}$ higher [59], which indicates that lysozyme is more stable with respect to unfolding than that of ribonuclease A. Thus, lysozyme is more likely to retain a more compact structure in unfavorable solution conditions. The deviations between the calculated and observed maximum charge states parallel the conformational stability of these proteins in solution and are consistent with ions that have similar conformation to those in solution upon entering the gas phase. The existence of different stable gas-phase conformers has been indicated by $\mathrm{H} / \mathrm{D}$ exchange [38, 39], collisional cross section $[60,61]$, and proton transfer experiments $[13,14,26]$.

High charge state ions have substantial Coulomb energy in the gas phase. For example, the $45+$ ion of carbonic anhydrase has a total Coulomb energy of 56 $\mathrm{eV}$. Thus, for very highly charged ions, the Coulomb repulsion is expected to be significantly greater than hydrophobic and hydrogen bond interactions [40]. This should result in unfolding of any tertiary structure. Thus, our modeling of high charge states of proteins from a denaturing solution as completely elongated linear strings appears reasonable. It should be noted that bending of the ion in the gas phase has negligible effects on our calculated GB ${ }^{\mathrm{ap}}$ [30]. It also should be possible to solvate the charges through interactions with adjacent polarizable side-chain residues or with backbone carbonyl oxygens without significantly effecting a primarily linear structure.

\section{Intramolecular Charge Solvation}

Values of $\mathrm{GB}_{\text {Intrinsic }}$ for various sites of protonation in the peptides and proteins are obtained from measurements of the GB of small peptides that contain only one basic residue. As a rough approximation, our model treats residues of a given type, for example, 
Lys, identically, independent of their local environment such as adjacent residues and conformation of the protein. Clearly, better values of $\mathrm{GB}_{\text {Intrinsic }}$ could be obtained by taking these factors into account. The most significant source of error in this approximation would be if, for the higher charge states, the charges are no longer intramolecularly solvated, but rather extend into vacuum to reduce the Coulomb energy. The $\mathrm{GB}_{\text {Intrinsic }}$ values for these high charge state ions would then overestimate the true value by $12-17 \mathrm{kcal} / \mathrm{mol}$. To determine if this is energetically favorable, cytochrome $c$ and lysozyme are modeled as strings with the charges displaced from the backbone line of center by $6 \AA$ in a staggered (or trans) configuration. The value of $6 \AA$ was chosen as an estimate of the maximum distance a charge could be displaced from the backbone based on the lengths of Arg and Lys side chains. Obviously, the value for Pro and Trp would be less than this, so our calculations should overestimate the effects of staggering the charge on lowering the Coulomb energy.

For cytochrome $c$, the lowest energy configuration of the $21+$ ion with the charges on the line of center (solvated) has an average total Coulomb energy of 513 $\mathrm{kcal} / \mathrm{mol}$, and the staggered structure has a value of $460 \mathrm{kcal} / \mathrm{mol}$. Thus, an average value of only 2.5 $\mathrm{kcal} / \mathrm{mol}$ of Coulomb energy is relieved per charge if solvated charges extend into vacuum in a staggered configuration. This value is $3.0 \mathrm{kcal} / \mathrm{mol}$ per charge for lysozyme. Effects on protonation sites that are closely spaced will be greater. In these ions, the maximum Coulomb energy relieved by displacement or "desolvation" of an individual proton is $8 \mathrm{kcal} / \mathrm{mol}$. This value is significantly less than the estimated stabilization energy possible through charge solvation (12-17 $\mathrm{kcal} / \mathrm{mol}$ ) [30], although the energy of charge solvation in a peptide may overestimate that in a fully elongated highly charged protein. Nevertheless, these calculations indicate that virtually all charges should be solvated in the gas phase and will not extend into vacuum.

The above calculations used a value of $\varepsilon_{r}=2.0$. We repeated these calculations for the $21+$ ion of cytochrome $c$ with $\varepsilon_{r}=1.0$ (the vacuum permittivity) to obtain an upper limit to the effects of Coulomb repul- sion. The lowest energy proton configurations were recalculated with this value and a total of six were obtained. For these configurations, we find an average decrease in Coulomb energy of $3.4 \mathrm{kcal} / \mathrm{mol}$ per charge for the all-trans versus the linear charge configuration. With increased Coulomb repulsion, greater energy is relieved by displacement or desolvation of individual protons. For 5 out of $126(=6 \times 21)$ total protons for which this is most significant, we find the Coulomb energy is lowered by $11-14 \mathrm{kcal} / \mathrm{mol}$. Thus, it is possible that some small fraction of protons in the maximum charge state may be unsolvated. However, it is unlikely that this number is significant.

\section{Where Charges Reside}

In many peptides and proteins, our calculated maximum charge state is similar to or the same as those predicted solely on the basis of number of basic residues (Arg, Lys, His, $\mathrm{N}$-terminus). However, the assigned proton configurations in these ions are usually different. For example, the maximum experimental charge state of ubiquitin is $13+$ [50]. This same value is predicted from the sum of basic residues. Our calculations indicate that the $13+$ ion has a GB app 0.4 $\mathrm{kcal} / \mathrm{mol}$ higher than the GB of methanol, so that the $14+$ ion should exist (eq 2), although its abundance is anticipated to be small. Even for the $13+$ ion, we find that not all the basic residues are protonated. A plot of the frequency of protonating individual residues as a function of charge state for ubiquitin is shown in Figure 2. For the $13+$ ion, our calculations indicate that Lys 29 is not protonated due to the close proximity to the lysines in positions 27 and 33. Pro 19, which is not very close to any basic site, becomes fully protonated at the $12+$ charge state. Similarly, Pro 37 and Pro 38 are protonated in a fraction of these proton configurations. In molecules such as carbonic anhydrase, which have fewer basic sites than the maximum charge state, protonation at the next most basic sites, Pro, Gln, and Trp, is favored. This is illustrated in Figure 3, which shows the frequency of protonation of individual sites in carbonic anhydrase as a function of charge state. Our calculations indicate that these sites begin to be protonated between charge states $18+$ and

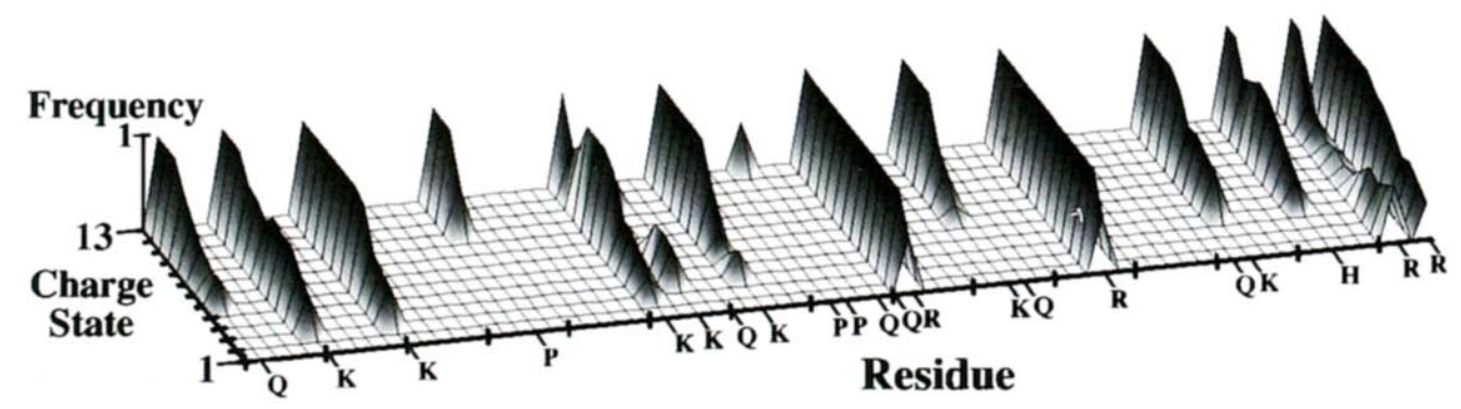

Figure 2. Frequency of protonating residues in denatured gas-phase ubiquitin as a function of charge state (basic amino acids are labeled with the single-letter codes; Arg, Lys, and His are shown in boldface). 


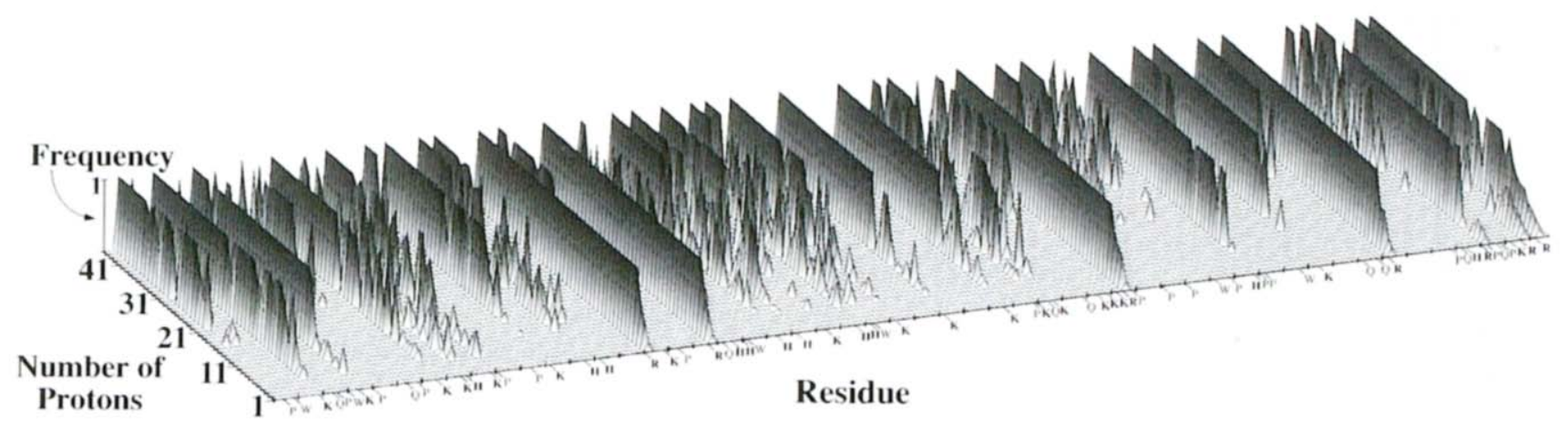

Figure 3. Frequency of protonating residues in denatured gas-phase bovine carbonic anhydrase as a function of charge state (basic amino acids are labeled with the single-letter codes; Arg, Lys, and His are shown in boldface).

$26+$, a range where nearly all Arg and between $35-60 \%$ of Lys and His are protonated.

The general trend of protonation of less basic sites even when more basic sites remain available is illustrated in Figure 4, which shows the average fraction of each basic site that is protonated as a function of the percentage of maximum charge state for four proteins: ubiquitin, cytochrome $c$ (denatured), lysozyme (denatured), and carbonic anhydrase. This shows that the contribution of the less basic residues Pro, Gln, and Trp is, on average, only significant when the charge state of the ion exceeds $60 \%$ of the maximum value. At this point, $\sim 60 \%$ of Lys and His and nearly all Arg residues are protonated. This trend reflects the values we use for $G B_{\text {Intrinsic }}$.

\section{Reactive Cross Sections}

The kinetics of proton transfer reactivity of multiply protonated protein ions to neutral bases is a topic of considerable interest [24, 27,62]. Using the Langevin model [63] and assuming all charge sites are identical, a collisional cross section that increases approximately linearly with the number of charges is predicted. How-

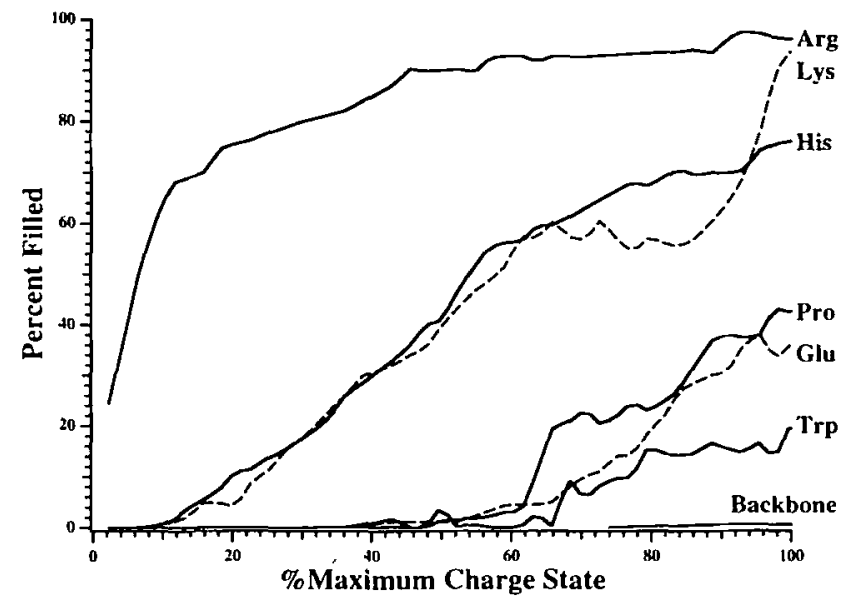

Figure 4. Average values for the fraction of basic residues protonated as a function of percent maximum charge state for carbonic anhydrase, cytochrome $c$, lysozyme, and ubiquitin. ever, proton transfer kinetics of isolated charge states of proteins do not appear to scale linearly with charge. In a typical protein, the basicity of different sites in the ion can differ dramatically, that is, some protons will be less reactive to proton transfer than others. Thus, the reactive cross section should be less than what would be predicted based on the collisional cross section when the GBapf of the ion is comparable to the $G B$ of the neutral base.

To calculate the reactive cross section for proton transfer for any charge state of a protein, we find the number of protonation sites in all the lowest energy proton configurations that have basicities within 3 $\mathrm{kcal} / \mathrm{mol}$ of the calculated $\mathrm{GB}^{\text {app }}$ for that charge state. We then divide this number by the total number of protonation sites and multiply by the charge state to give a reduced cross section. For example, the $20+$ charge state of cytochrome $c$, with five lowest energy configurations, has a $\mathrm{CB}^{\mathrm{app}}$ of $177 \mathrm{kcal} / \mathrm{mol}$. Only one proton in each of these configurations has a GB within $3 \mathrm{kcal} / \mathrm{mol}$ of this value. Thus, the reactive cross section for proton transfer from the $21+$ charge state of cytochrome $c$ to a neutral reference base with a GB $\sim 177 \mathrm{kcal} / \mathrm{mol}$ would be expected to be approximately the same as that of a singly charged ion with a base of comparable basicity.

The reduced reactive cross sections for carbonic anhydrase, cytochrome $c$, lysozyme, and ubiquitin as a function of charge are shown in Figure 5. Significant deviation from the Langevin cross section is observed for the high charge states. For these three proteins, the reduced cross section ranges between 1 and 6 , even for ions with as many as 35 charges. These results indicate that measurements of proton transfer kinetics of highly charged ions will reflect the chemistry of only the most reactive sites in the ion.

\section{Conclusions}

For the commonly electrosprayed proteins listed in Table 2, we find our calculated maximum charge states are on average within $6 \%$ of the experimental value. The sum of basic residues in each protein differs from the experimental maximum charge state by an average 


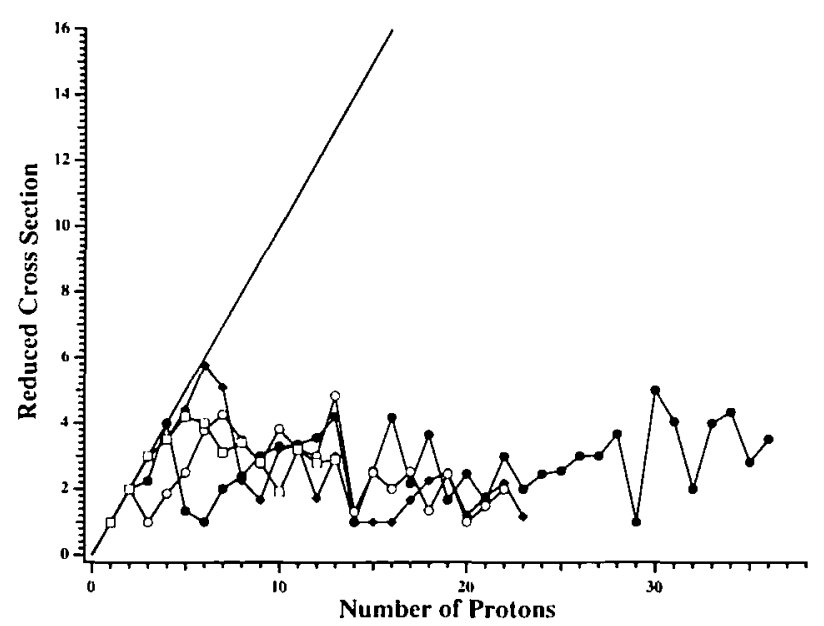

Figure 5. Calculated reduced cross section for proton transfer as a function of charge state from carbonic anhydrase (O), cytochrome $c(O)$, and lysozyme $(\$)$ to a neutral base that has a gas-phase basicity approximately equal to the apparent gas-phase basicity of the given charge state of the protein ion. The dashed line indicates the Langevin collisional reduced cross section. All cross sections are relative to that of a singly protonated ion reacting with a base of comparable gas-phase basicity

of $15 \%$ for these same proteins. These results indicate that the maximum charge state of proteins formed by electrospray ionization is determined by gas-phase reactions of these ions with solvent molecules from the electrospray process. For proteins for which there are fewer available reference spectra, we find our calculated values are usually greater than the highest reported experimental value. It is possible that the maximum extent of protonation has not yet been observed for these molecules. For peptides with many basic sites, the calculated gas-phase reactivity accurately accounts for the maximum charge state. In contrast, the maximum charge state of peptides with few basic sites appears to correlate to the number of residues that are protonated in solution.

A systematic study of the maximum extent of protonation observable for proteins and peptides electrosprayed from solvents of varied GB should provide evidence for this model. However, it would need to be undertaken on an instrument with which the maximum charge states are consistently observed. Effects of solution-phase chemistry, as well as other factors, would need to be carefully considered because they could obfuscate these results.

A number of factors contribute to the actual charge state distribution observed in ESI mass spectra. Gasphase reactions with bases added in solution or the gas phase can change these distributions [20-22, 24-31]. However, the role of gas-phase chemistry in the formation of conventional ESI distributions is less clear. Ions that have high internal and/or kinetic energy are more reactive to proton transfer; reaction of these ions with solvent molecules would produce lower charge state ions. In addition, solvent clusters are more basic than individual solvent molecules. Proton transfer to these more basic clusters would also result in the formation of lower charge state ions. It is interesting to note that significant abundance of protonated solvent is not typically observed. This indicates that gas-phase reactions are not likely to be the principal mechanism in the formation of the observed charge distribution, although instrumental factors, such as transmission efficiency, may contribute to the low abundance of these small ions.

Our calculations indicate that a significant fraction of less basic residues (Pro, Trp, and GIn) are protonated in high charge state ions. Protonation of backbone sites occurs, but is generally unfavorable. Only a few percent of all backbone sites are protonated even in high charge state ions. We find that the reactivity of individual protonated residues in a multiply protonated protein can vary dramatically. In high charge state ions, only a small fraction of the protons have calculated $\mathrm{GB}^{\text {app }}$ within $3 \mathrm{kcal} / \mathrm{mol}$ of the most reactive proton. From these values, the reduced cross section for proton transfer can be calculated. This value is found to be significantly lower than the Langevin collision frequency for high charge state ions.

We are investigating factors that should improve the accuracy of this model. For example, the $\mathrm{CB}_{\text {Intrminic }}$ values could be improved by taking into account the local environment of a residue, which include effects of adjacent residues as well as the overall conformation of the protein. We also are investigating the application of our model to negatively charged ions and the prediction of fragmentation products of multiply charged ions.

\section{Acknowledgments}

The authors acknowledge W. D. Price for helpful discussions, thank G. D. Brayer for generously providing the x-ray crystal structure coordinates for equine cytochrome $c$, and acknowledge generous financial support from the Arnold and Mabel Beckman Foundation (M1652), the National Science Foundation (CHE9258178), the Analytical Chemists of Pittsburgh (M1601), the Exxon Foundation (1360)5), and Finnigan MAT through sponsorship of the 1994 American Society for Mass Spectrometry Research Award (E.R.W.).

\section{References}

1. Fenn, J. B.; Mann, M.; Meng, C. K.; Whitchouse, C. M. Scionce 1989, 246, 64-71.

2. Smith, R. D.; Loo, 1. A.; Ogorzalek Loo, R. R.; Busman, M.; Udseth, H. R. Mass Spictrom. Rer. 1991, 10, 359-452.

3. Beu, S. C.; Senko, M. W.; Quinn, I. P.; MeLafferty, F. W. 1. Amr. Soc. Marss Spectrom. 1993, 4, 19(1-192.

4. Nohmi, T.; Fenn, J. B. I. Am. Ch'm. Soc. 1992, 114, 3241-3240.

5. Fenn, J. B. I. Am. Soc. Miss Spetrom. 1993, 4, 524-535.

6. Dole, M.; Mach, L. L.; Hines, R. L.; Mobley, R. C.; Ferguson, L. P.; Alice, M. B. J. Ch''m. Physs. 1968, 49, 2240-2249.

7. Schmelzeisen-Redecker, G.; Bütfering, L.; Röllgen, F. W. IIIt. 1. Mass Spectrom. Lon Processes 1989, 90, 139-150.

8. Iribarne, J. V.; Thomson, B. A. I. Ch'm. Physs. 1976, 6t, 2287-2294.

9. Kebarle, P.; Tang, L. Amal. Ch'm. 1993, 6.5, 972A-986A. 
10. Covey, T. R, Bonner, R. F.; Shushan, B: I; Henion, J. Rapid Commuin. Mass Spectrom. 1988, 2, 249-256.

11. Chowdhury, S. K.; Katta, V.; Chait, B. T. J. Am. Chem. Soc: $1990,112,9012-9013$.

12. Mirza, U.; Cohen, S.; Chait, B. T. Anal. Chem. 1993, 65, 1-6.

13. Loo, J. A.; Edmonds, C. G.; Udseth, H. R.; Smith, R. D. Anal. Chem. 1990, 62, 693-698.

14. Loo, J: A.; Ogorzalek Loo, R. R.; Udseth, H. R.; Edmonds, C. G.; Smith, R. D. Rapid Commun. Mass Spectrom. 1991, 5, 101-105.

15. Guevremont, R.; Siu, K. W. M.; LeBlanc, J. C. Y.; Berman, S. S. I. Am. Soc. Mass Spectrom, 1992, 3, 216-224.

16. LeBlanc, J. C. Y.; Siu, K. M.; Guevremont, R. Anal. Chem. $1994,66,3289-3296$.

17. Kelly, M. A.; Vestling, M. M.; Fenselau, C. C.; Smith, P. B. Org. Mass Spectrom. 1992, 27, 1143-1147.

18. Ashton, D. S.; Beddell, C. R.; Cooper, D. J.; Green, B. N.; Oliver, R. W. A. Org. Mass Spectront. 1993, 28, 721-728.

19. Tang, L; Kebarle, P. Anal. Chem. 1993, 65, 3654-3666.

20. LeBlanc, J. C. Y.; Wang, J.; Guevremont, R.; Siu, K. W. M. Org. Mass Spectrom. 1994, 29, 587-593.

21. Wang, G.; Cole, R. B. Org. Mass Spectrom. 1994, 29, 419-427.

22. Ogorzalek Loo, R. R., Smith, R. D. J. Mass Spectrom. 1995; 30 , 339-347:

23. Gulcicek, E. E.; Shen, S.; Boyle, J. D.; Whitehouse, C. M.; Harrison, D. H.; Moore, P. B. Proceedings of the 39tli ASMS Conference on Mass Spectrometry and Allied Topics; Nashville, TN, 1991; pp 1245-1246.

24. McLuckey, S. A.; Van Berkel, G. J.; Glish, G. L. J. Am. Chem. Soc: $1990,112,5668-5670$.

25. Cassady, C. J.; Wronka, J.; Laukien, F. H. Rapid Commin. Mass Spectrom. 1994, 8, 394-400.

26. Ogorzalek Loo, R. R.; Smith, R. D. J. Am. Soc. Mass Spectrom. $1994,5,207-220$.

27. Ogorzalek Loo, R. R., Winger, B. E.; Smith, R. D. J. Am. Soc. Mass Spectrom. 1994, 5, 1064-1071.

28. Gross, D. S.; Williams, E. R. J. Am. Chem. Soc. 1995, 117, $883-890$.

29. Gross, D. S; Rodriguez-Cruz, S. E.; Bock, S.; Williams, E. R. I. Pliys, Chient: 1995, 99, 4034-4038

30. Schnier, P. D.; Gross, D. S.; Williams, E. R. J. Am. Chem. Soc. $1995,117,6747-6757$.

31. Williams, E. R.; Gross, D. S.; Schnier, P. D.; Rodriguez-Cruz, S. E.; Fagerquist, C. K. Proceedings of the 43rd ASMS Conference on Mass Spectrometry and Allied Topics; Atlanta, GA, May $21-26,1995$; TOC 11:50.

32. Aue, D. H.; Bowers, M. T. In Gas-Phase Ion Chemistry, Vol. 2; Bowers, M. T., Ed.; New York: Academic Press, 1979; Chap. 9.

33. DeFrees, D. J.; Mclver, R. T.; Hehre, W. J. J. Am. Chem. Soc. $198,102,3334-3338$.

34. Meluckey, S. A.; Cameron, D.; Cooks; R. G. I. Am. Chem. Soc. 1981, 103, 1313-1317.

35. Javahery, G.; Petrie, S.; Wincel, $\mathrm{H}_{\text {; }}$ Wang, J; Bolime; D. K. J. Am. Chem. Soc. 1993, 115, 6295-6301.
36. Bursey, M. M.; Pederson, L. G. Org. Mass Spectrom. 1992, 27, 974-975.

37. Petrie, S.; Javahery, G; Wincel, H; Wang, J.; Bohme, D. K J. Am. Chem. Soc. 1993, 115, 6290-6294.

38. Suckau, D; Shi, Y; Beu, S. C.; Senko, M. W.; Quirn, J. P.; Wampler, F. W.; McLafferty, F. W. Proc. Nat'l. Acad. Sci. U.S.A. 1993, 90, 790-793.

39. Wood, T. D; Chorush, R. A.; Wampler, F. M.; Little, D. P.; O'Connor, P: B.; McLafferty, F. W. Proc. Nat $\%$. Acad. Sci. U.S.A. 1995, 92, 2451-2454.

40. Creighton, T. E. Proteins, 2nd ed; New York: W. H. Freeman and $C_{0}, 1993$.

41. Rockwood, A. L.; Busman, M.; Smith, R. D. Int. I. Mass Spectrom. Ion Processes 1991, 111, 103-129.

42. Wu, J.; Lebrilla, C. B. J. Am: Chem. Soc. 1993, 115, 3270-3275.

43. Wu, J.; Lebrilla, C. B. I. Am. Soc. Mass Spectrom. 1995, 6, 91-101.

44. Wu, Z; Fenselau, C. I. Am. Soc. Mass Spectrom, 1992, 3, 863-866.

45. Gorman, G. S.; Amster, I. J. J. Ani. Chem. Soc. 1993, 115, 5729-5735.

46. Wu, Z.; Fenselau, C. Rapid Commun. Mass Spectrom. 1992, 6 , $403-405$.

47. Wu, Z.; Fenselau, C. Rapid Commun. Mass Spectrom. 1994, 8 , $777-780$.

48. Lias, S. G.; Liebman, J. F.; Levin, R. D. I. Phys. Chem. Ref. Datin 1984, 13, 695-808.

49. Wu, Z.; Fenselau, C. Tetrahedron 1993, 49, 9197-9206.

50. Katta, V,; Chait, B. T. Rapid Commun. Mass Spectioni. 1991, 5 , 214-217.

51. Kirkpatrick, C. D.; Gelatt, C. D.; Vecchi, M. P. Science 1983 220, 67i-680.

52. Press, W. H.; Flannery, B. P.; Teukolsky, S. A.; Vetterling, W. T. Numerical Recipes in Pascal; Cambridge, U.K.: Cambridge University Press, 1989.

53. Wan Abdullah, W. A. T. J. Comp. Pliys. 1994, 110, 320-326.

54. Lide, D. R., Ed. CRC Handbook of Chemistry and Physics; Boca Raton, FL: CRC Press, 1993:

55. Chernushevich, I. V.; Ens, W.; Standing, K. G. Proceedings of the 43rd ASMS Conference on Mass Spectronetry and Allied Topics; Allanta, GA, May 21-26, 1995; FOD 10:30.

56. Loo, J. A.; Ogorzalek Loo, R. R.; Udseth, H. R.; Fulton, J. L.; Smith, R. D. Rapid Communn. Mass Spectrom. 1992, 6, 159-165

57. Buck, M.; Radford, S. E.; Dobson, C. M. Biochemistry 1993, 32 669-678.

58. Schwarz, F. P, Kirchhoff, W. H. Thermochint. Actn 1988, 128 267-275.

59. Schwarz, F. P. Thermochint. Actn 1989, 147, 71-91.

60. Covey, T.; Douglas, D. J. J. Am. Soc. Miss Spectrom. 1993, 4, 616-623.

61. Cox, K. A.; Julian, R. K.; Cooks, R, G.; Kaiser, R. E. J. Am. Soc. Mass Spectrom. 1994; 5, 127-136.

62. Ikonomou, M. G.; Kebarle, P. Int. I. Mass Spectrom. Ion Processes 1992, 117, 283-298.

63. Langevin, P. Ann Chim. Plyss 1905, 5, 245. 